\title{
Fine Coregistration \& Brunch-cut for Unwrapping Interferometric Phase
}

\author{
Hadj Sahraoui Omar \\ 5 HP RM6HQMQI IGHDUN HQWS OHUD \\ 6SLFH\$ J HDF MIIS YHOXHGH(D)

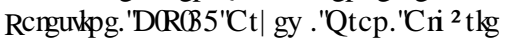 \\ Sahraoui_omar1@yahoo.fr
}

\author{
Berrichi Faouzi \\ 5 H RMG6HOMQ IGHDWA HQWS OHIDQ \\ 6SDFH\$ J HQF MIIS YHQXHGH(D)

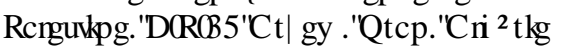 \\ fberrichi@cts.asal.dz,
}

\author{
Chamakhi Djemoui

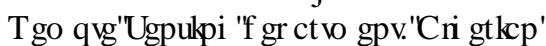 \\ 6SDFH\$ J HPF WI

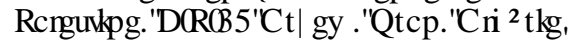 \\ dchamakhi@cts.asal.dz
}

\begin{abstract}
Earth observation and environmental control are becoming more important where the realization of DEM by the technique of interferometry of RSO images facilitates the control of terrestrial deformations, which is the subject of this paper.The unwrapping phase is a very important step in the interferometry process, especially to treat it carefully in steep degraded and mountainous ground. We have focused on the reduction and sometimes the elimination of the residues in flat terrains and otherwise to keep the maximum of the residues in the very steep sites. For this purpose, the methods developed and proposed are based on a transformation by the ISODATA which is based on KMEANS and the double passage of the median filter, and this in order to limit the creation of the brunch-Cut for the progress of the filtered interferogram (elimination of large black spot on The Brunch-Cut image).The tests of this method were carried out on several sites, and showed good results.
\end{abstract}

Keywords-SAR Interferometry, Unwrapping phase, ISODATA, residues, brunch-cut;

\section{SAR INTERFEROMETRY FOR DEM} GENERATION:

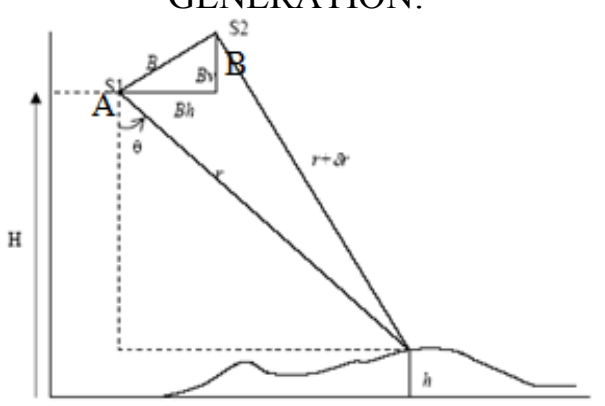

Figure 1. InSAR Geometry
After registration process, the complex interferograms are formed by multiplying each complex pixel of the first image by the complex conjugate of the same pixel in the second image. [7] the phase difference $\phi$ between two acquisitions signals related the same object. It's calculated by the following expression:

$\psi(i, j)=A(i, j) B^{*}(i, j) \mid=\rho_{A}(i, j) \rho_{B}(i, j) e^{j\left(\phi_{A}(i, j)-\phi_{B}(i, j)\right)}$

Where $\left(A \mathrm{~S}_{1}, \mathrm{~B} \mathrm{~S}_{2}\right)$ are the two master and slave images and $\left(\rho_{A}, \rho_{B}\right)\left(\phi_{A}, \phi_{B}\right)$ are respectively the modules and the arguments of the images $\mathrm{A}$ and $\mathrm{B}$. and developed by the expression With:

$\phi=\frac{4 \pi(\delta r)}{\lambda}=\frac{4 \pi\left(B_{h} \sin \theta-B_{v} \cos \theta\right)}{\lambda}$

$(\boldsymbol{\lambda}$ : the wavelength, $\boldsymbol{\delta} \boldsymbol{r}$ : Range difference, $\boldsymbol{B} \boldsymbol{h}$ : Horizontal baseline, $\boldsymbol{B} \boldsymbol{v}$ : Vertical baseline, $\boldsymbol{\theta}$ : look angle).

Based on the look angle and range difference changes, the interferogram generated as:

$\Delta h=\frac{\lambda r \sin \theta}{4 \pi B} \Delta \phi$

$r$ : Range of image A, $\Delta \phi:$ Phase between $\mathrm{A}$ and $\mathrm{B}$,

$B$ : Base line(normal)

The description of Interferometry chains is given by this algorithm:

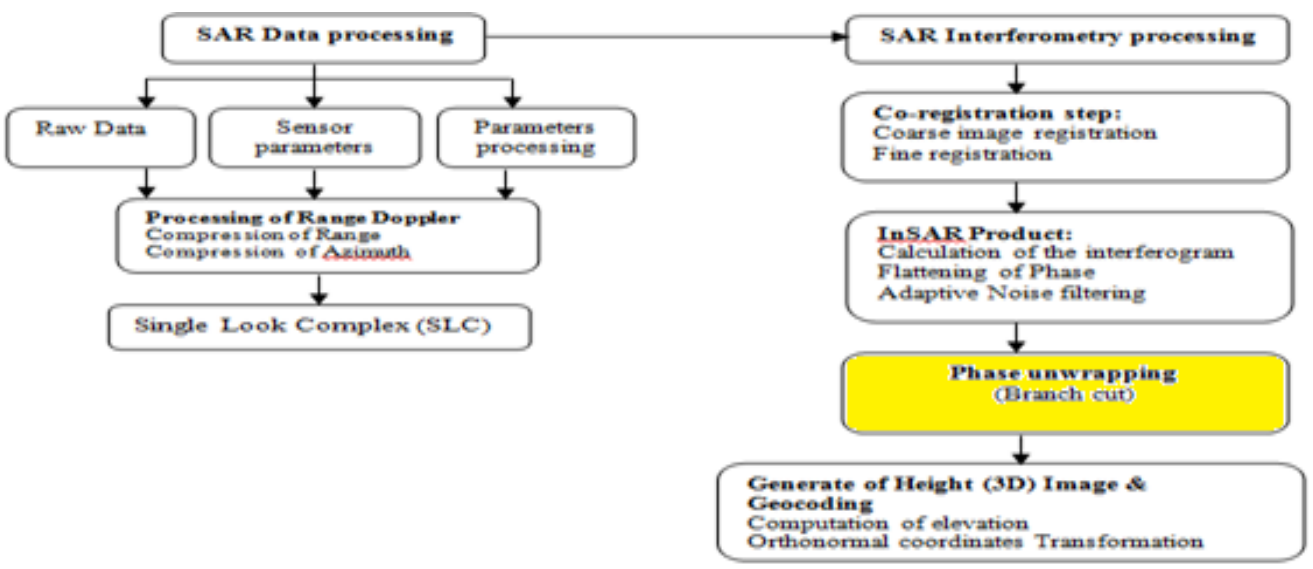

Figure 2. InSAR processing organization. 


\section{A. InSAR processing:}

Step 1:Image Co-registration: the phase difference and consistency are defined from the normalized correlation[6];[8];[3]:

$C=\rho e^{j \phi}=\frac{E\left\{z_{M} z_{S}^{*}\right\}}{\sqrt{E\left\{\left|z_{M}\right|^{2}\right\} E\left\{\left|z_{S}\right|^{2}\right\}}}$

We interested to the fine co-registration after a global registration, to estimate the error. With different equations[9];[1]; [2]:

$$
\begin{aligned}
& \varepsilon_{\text {recalage }}=\frac{\delta\left(R_{2}-R_{1}\right)}{\delta d}-\frac{\overline{\delta\left(R_{2}-R_{1}\right)}}{\delta d} \approx \\
& \frac{1}{k_{\text {st }}} \frac{b}{R}\left(\frac{\cos \left(\theta-\alpha_{b}\right)}{\tan \left(\theta-\alpha_{q}\right)}-\frac{\cos \left(\theta-\alpha_{b}\right)}{\tan (\theta)}\right) \\
& \varepsilon_{\text {recalage }} \approx \frac{1}{k_{\text {st }}} \frac{b}{R} \cos \left(\theta-\alpha_{b}\right)\left(\frac{\cos \left(\theta-\alpha_{q}\right)}{\sin \left(\theta-\alpha_{q}\right)}-\frac{\cos (\theta)}{\sin (\theta)}\right) \\
& \varepsilon_{\text {recalage }} \approx \\
& \frac{1}{k_{\text {st }}} \frac{b}{R} \cos \left(\theta-\alpha_{b}\right) \frac{\sin (\theta) \cos \left(\theta-\alpha_{q}\right)-\cos (\theta) \sin \left(\theta-\alpha_{q}\right)}{\sin (\theta) \sin \left(\theta-\alpha_{q}\right)} \\
& \varepsilon_{\text {recalage }} \approx \frac{1}{k_{\text {st }}} \frac{b}{R} \cos \left(\theta-\alpha_{b}\right) \frac{\sin \left(\alpha_{q}\right)}{\sin (\theta) \sin \left(\theta-\alpha_{q}\right)}
\end{aligned}
$$

Step 2:Interferogram generation: the interferometer is produced by the multiplication of each selected pixel of the first SLC image by the second conjugate of the same pixel, where the coherence $\rho$ is related to the normalized correlation of the intensities [4]; [5]

$$
\rho= \begin{cases}\sqrt{2 \gamma_{I}-1} & \gamma_{I} \geq 1 / 2 \\ 0 & \gamma_{I}<1 / 2\end{cases}
$$

With:

$$
\gamma_{I}=\frac{\sum_{i=1}^{L}\left|u_{1}(i)\right|^{2}\left|u_{2}(i)\right|^{2}}{\sqrt{\sum_{i=1}^{L}\left|u_{1}(i)\right|^{4} \sum_{i=1}^{L}\left|u_{2}(i)\right|^{4}}}
$$

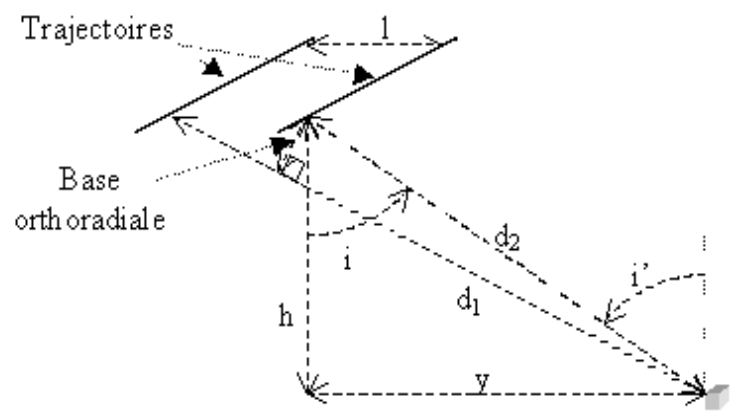

Figure 3. Calculating the phase shift InSAR

B. Calculation of the unwrapping phase:

With figure 3, we get:

$\Delta \phi=2 \frac{2 \pi}{\lambda}\left(d_{2}-d_{1}\right)$
Or,

$d_{1}=\sqrt{h^{2}+(y+l)^{2}}=\sqrt{h^{2}+y^{2}+2 y l+l^{2}}$

$=\sqrt{h^{2}+y^{2}} \cdot \sqrt{1+\frac{2 y l+l^{2}}{h^{2}+y^{2}}}=d_{2} \cdot \sqrt{1+\frac{2 y l+l^{2}}{d_{2}{ }^{2}}}$

And $\quad d_{2}=\sqrt{h^{2}+y^{2}}$

In first order:

$d_{1} \approx d_{2}\left(1+\frac{y l}{d_{2}^{2}}\right) \Rightarrow \Delta \phi \approx \frac{4 \pi}{\lambda} \frac{y l}{d_{2}}=\frac{4 \pi}{\lambda} l\left(\sin \left(i^{\prime}\right)\right)$

(Where $\mathrm{i}=\mathrm{i}$ ' positive)

By replacing the orthoradial base $\boldsymbol{B}_{\text {ortho }}$ at the equation 11:

$l=\frac{B_{\text {ortho }}}{\cos (i)} \longrightarrow \Delta \phi \frac{4 \pi}{\lambda} \tan (i) B_{\text {ortho }}$

In this case, the local impact $i^{\prime}$ is different $i$ tilt, with:

$i^{\prime}=\arcsin \left(\frac{R_{T}+h}{R_{T}} \sin (i)\right)$

\section{Variations of the phase shift}

To extract the relief, we're going to translate this variation $\Delta 2 f$ in terms of changes in positions. For this we need to start from the equation 11, since the orthoradial base varies depending on $\mathbf{Y}$.

$\partial(\Delta \phi)=\frac{4 \pi}{\lambda} l \cdot \partial\left(\frac{y}{d}\right)=\frac{4 \pi}{\lambda} l \cdot \frac{\partial y \cdot d-y \cdot \partial d}{d^{2}}=\frac{4 \pi}{\lambda} \frac{l}{d} \cdot[\partial y-\partial d \sin (i)]$

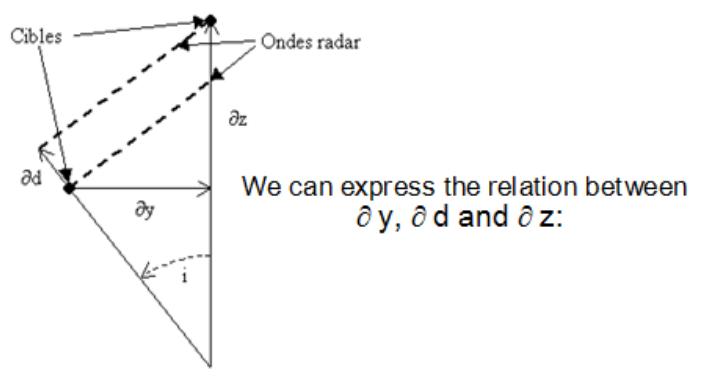

Figure 4. Coordinates of two infinitely close targets.

$$
\text { where: } \begin{aligned}
\partial(\Delta \phi)=\frac{4 \pi}{\lambda}, \frac{l}{d} & {\left[\left(\frac{\partial z}{\tan (i)}+\frac{\partial d}{\sin (i)}\right)-\partial d \sin (i)\right] } \\
& =\frac{4 \pi}{\lambda}, \frac{B_{\text {ombe }}}{d} \cdot \frac{1}{\cos (i)}\left[\frac{\partial z}{\tan (i)}+\partial d \frac{1-\sin ^{2}(i)}{\sin (i)}\right]
\end{aligned}
$$

Finally: $\Delta^{2} \phi=\frac{4 \pi B_{\text {onte }}}{\lambda \cdot d}\left[\frac{\Delta z}{\sin (i)}+\frac{\Delta d}{\tan (i)}\right]$
(10)

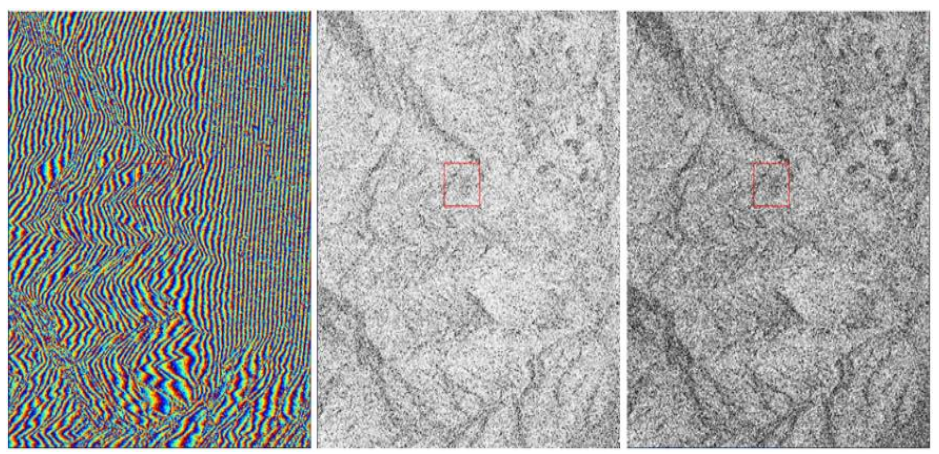

Figure 5: result of co-registration and Interferogram generation. 


\section{Orbital fringes:}

Influences other than surface deformation are contained in the difference in phase between two observations, is called orbital fringe and has roots in the difference between two orbits. In fact, the phase differences calculated (equation 15) correspond to the topography in the plane perpendicular to the direction of view. If the orbits are parallel, this corresponds to a ramp of phase in distance, giving visually a suite of parallel to the trajectory fringes spaced fairly regularly in distance, is that it represents the Figure 5 (in the left image): so subtract the second term of equation 15 to get the elevation $\Delta z$, it is shown in Figure 6.

$\Delta^{2} \phi=\frac{4 \pi B_{\text {ortho }}}{\lambda \cdot d}\left[\frac{\Delta z}{\sin (i)}+\frac{1}{\text { t }} 1(2)\right] \Rightarrow \Delta^{2} \phi=\frac{4 \pi B_{\text {ortho }}}{\lambda \cdot d}\left[\frac{\Delta z}{\sin (i)}\right]$

$\Delta^{2} \phi=\frac{4 \pi B_{\text {ortho }}}{\lambda \cdot d} \frac{\Delta z}{\sin (i)}$
Is that expression which is sometimes used to quantify the relief, the base orthoradiale is then taken as constant, however the exact expression is as follows:

$\Delta^{2} \phi=\frac{4 \pi l}{\lambda \cdot d} \frac{\Delta z}{\tan (i)}$

Of course, ' $i$ ' is the inclination of the satellite; even if we want to use the local incidence, taking into account the rotundity of the Earth, it is necessary to use the equation 13. The orbital position does not affect the amplitude and coherence images (Images in the Right of figure 5 and 6). They remain identical in Figures 5 and 6.

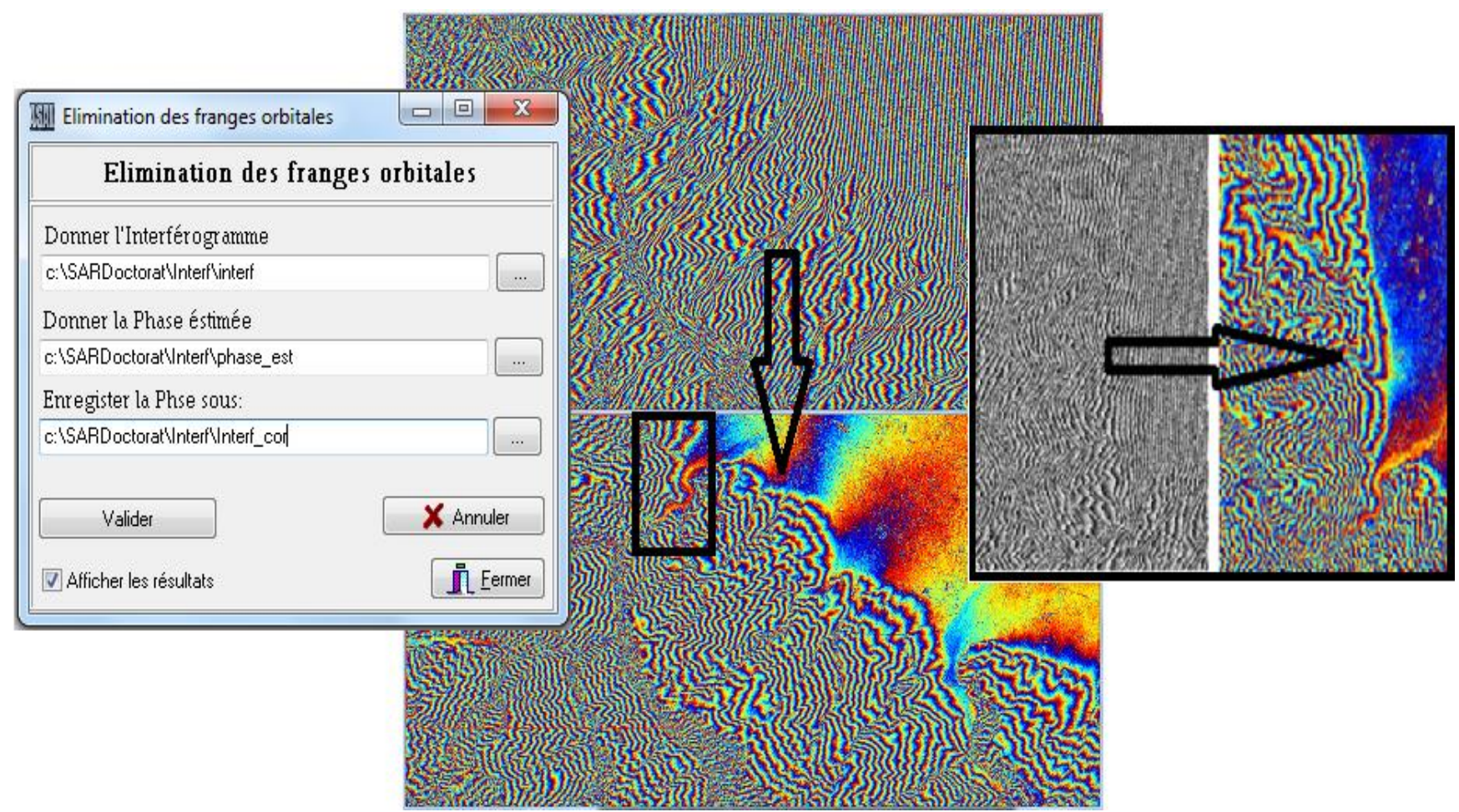

Figure 6. Elimination of orbital fringes

In this figure it can be seen that the phase image (Image on the left of figure 6) has completely changed in comparison with that of Figure 5. It is clearly explained that all the orbital fringes are eliminated, and only the topographical fringes are retained

\section{METHOD BASED ON ISODATA FOR UNWRAPPING PHASE:}

This method consists of the combination of several treatments:

-The median filter of size $5 \times 5$ is applied twice, one after the other; -Then classify the pixels in order to segment into regions using the Isodata method.
We will classify the image in two parts:

-1 / the background which symbolizes the ground, and where the amplitude is homogeneous because there are no disturbing asperities.

$-2 /$ the regions corresponding to either areas of overlap or ground deformation.

\section{A. Presentation of the results:}

The application of the two median filters $5 * 5$ lets standardize grayscale pixels forming regions of the flat and mountainous deformed ground. 


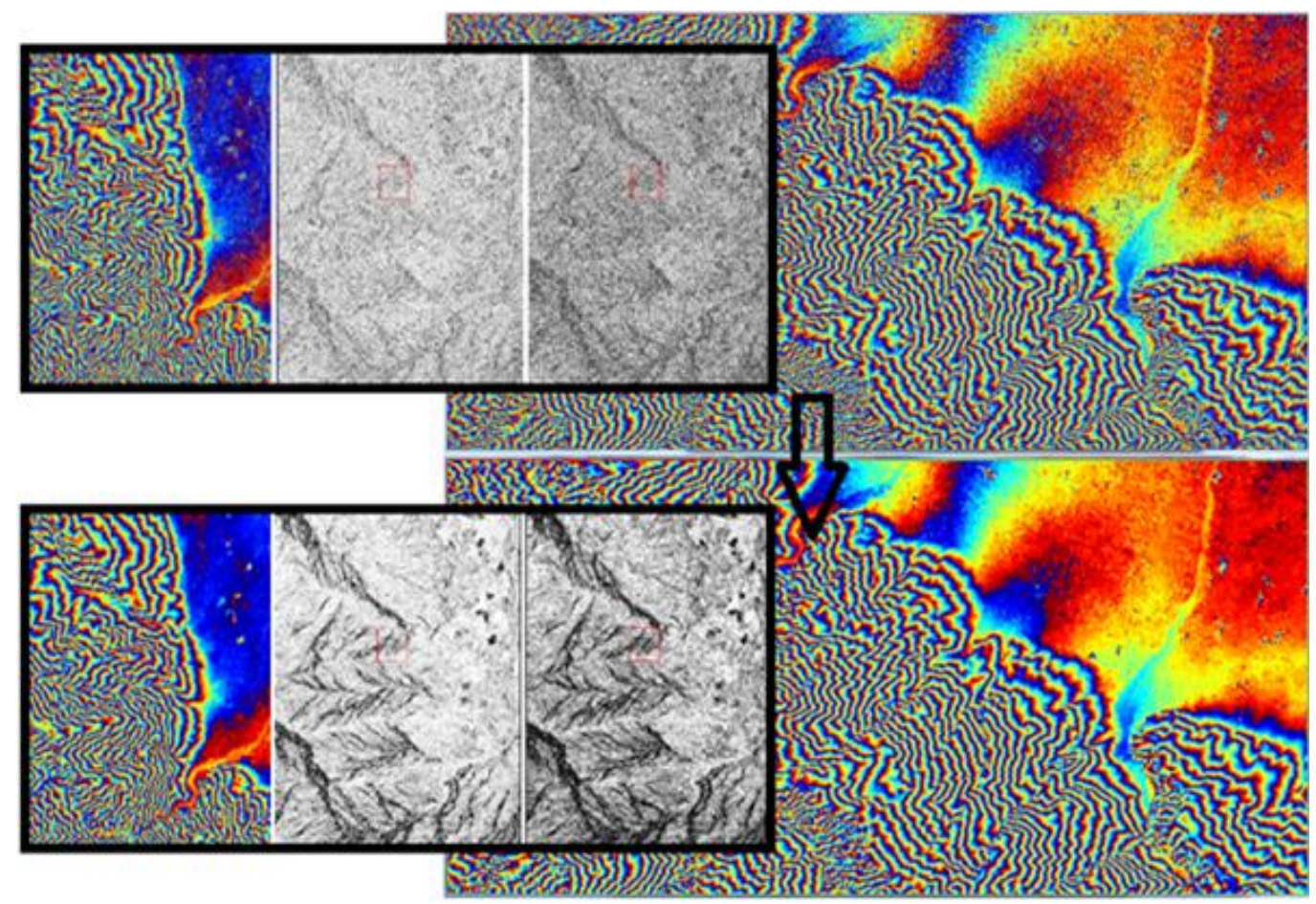

Figure 7. Aapplying the filter on the interferogram for the ISODATA application.

Image smoothing is an important operation used to attenuate a noise that corrupts information. This operation consists in applying to the images of Figure 6. It is clearly noticeable in Figure 7, noise reduction by smoothing, which will lead us directly to the application of the method ISODATA.

In the left image in figure 8, the overlap area was well isolated. In the right image, the application of the improved classification process leads to the obtaining of the boundaries of the deformed ground. It may be noted that they are not straight, contrary to what we see with eye on the magnitude image. it can then assume that some of the pixels forming the discontinuity will be forgotten. But the thickness of the frontier will certainly correct this error.

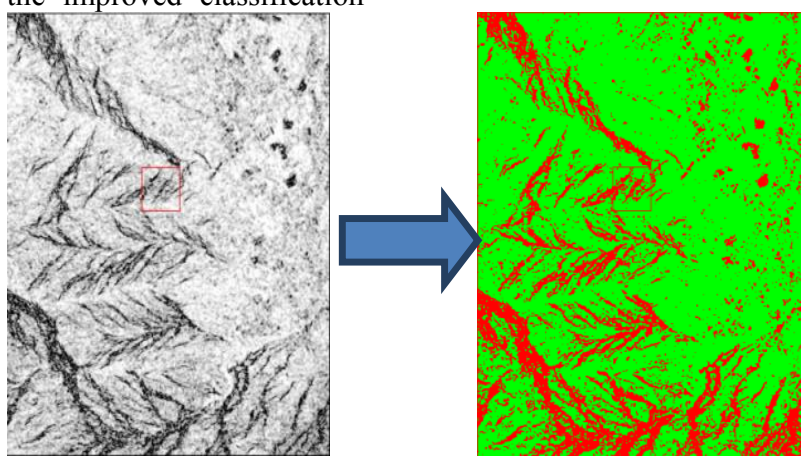

Figure 8. ISODATA applied to the interferogram magnitude image.

\section{B. Calculation of residue image for generation of the Brunch-Cut:}

The application can be accomplished by evaluating the sum of the derivatives along a closed path of $2 \times 2$ pixels. If this sum is equal to 1 or -1 , so-called positive or negative residual values respectively, it shows an incoherence of one of the 4 pixels. If the sum is zero it is said that the pixels of the borrowed path are not noisy.The application of Brunch-Cut extraction from the residues image based on the filtered interferogram image (before ISODATA application) does not have a good result because it eliminates large areas, especially in deformed ground and mountainous areas, which are presented by large black spots, which will eventually distort the course of phase as illustrated in the following figure 9 .

Quantization of the discontinuity of the unwrapping phase consists of calculating the pseudo-gradients on the shortest closed path [10]. On a regular grid such as the interferogram image. the computation of a residue is carried out on a node of $2 \times 2$ pixels by adding the difference of the phases. It is expressed by the following equation:

$$
\operatorname{residu}(\mathrm{i} ; \mathrm{j})=\operatorname{gx}(\mathrm{i} ; \mathrm{j})-g y(\mathrm{i}+1 ; \mathrm{j})-\mathrm{gx}(\mathrm{i} ; \mathrm{j}+1)+g y(\mathrm{i} ; \mathrm{j})
$$

Calculation graph of residues

Once the residuals are calculated, Goldstein's method consists in linking the residues of opposite signs, assuming that a noise on the interferogram can generate a pair of residuals $(+1$ and -1$)$. The shortest lines connecting the residues are called cuts. The ends of the cuts are connected to the edges of the image if the sum in a cut 
is not zero (if the number of positive residues differs from the number of negative residues).

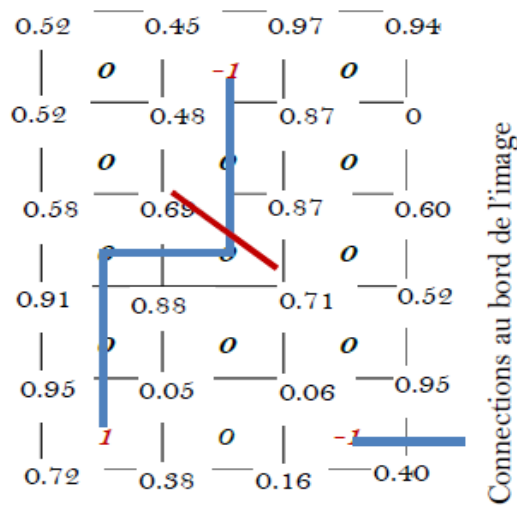

Graphe of residu connexion independent of the path of the chosen course, just avoid crossing a cut (blue line in Graphe of residu connexion). The figure 12 shows the result of brunch-cut unwrapping with avoidance of discontinuities.

When the residues are very close at the fringe transitions. Consequently, the more the fringes are close to each other, so the number of residues increases. A step of ISODATA on the interferogram is therefore necessary before the unwinding of the phase (figure9, 10, 11 and 12).

When all the residues are connected and the cuts are drawn

(Graphe of residu connexion), the unwrapping phase becomes

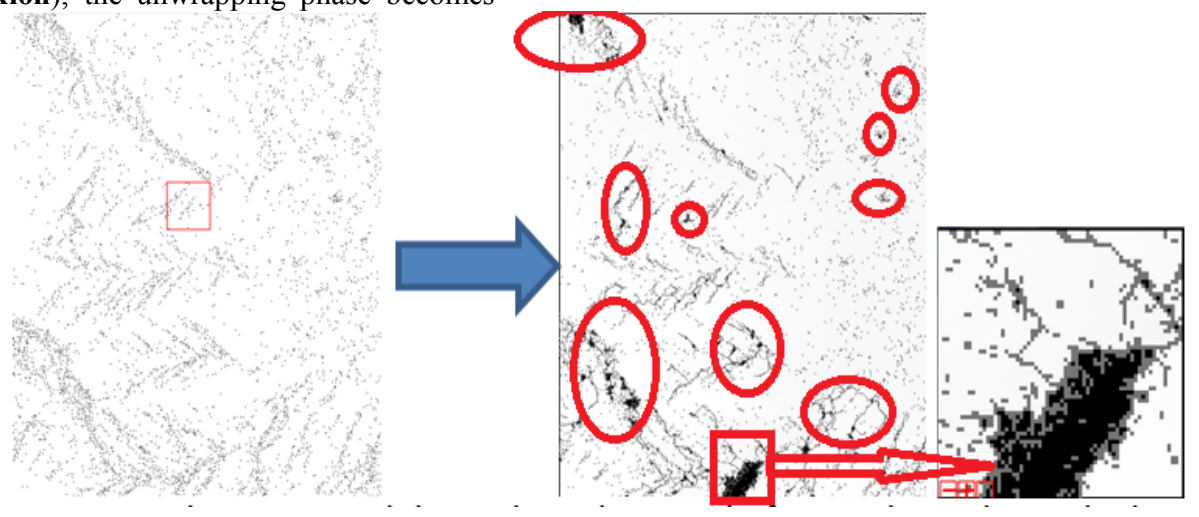

Figure 9. Residue image with the brunch-cut image before and after applying the method ISODATA.

To eliminate areas of conflicts during generation of the Brunch-Cut by the application of the residue, we merge the residues image and ISODATA image, to delineate areas that are affected by the unwrapping phase, which can be seen on figure 10 according to the points of the residues on the green areas of images classified by ISODATA and filtered Interferogram image.

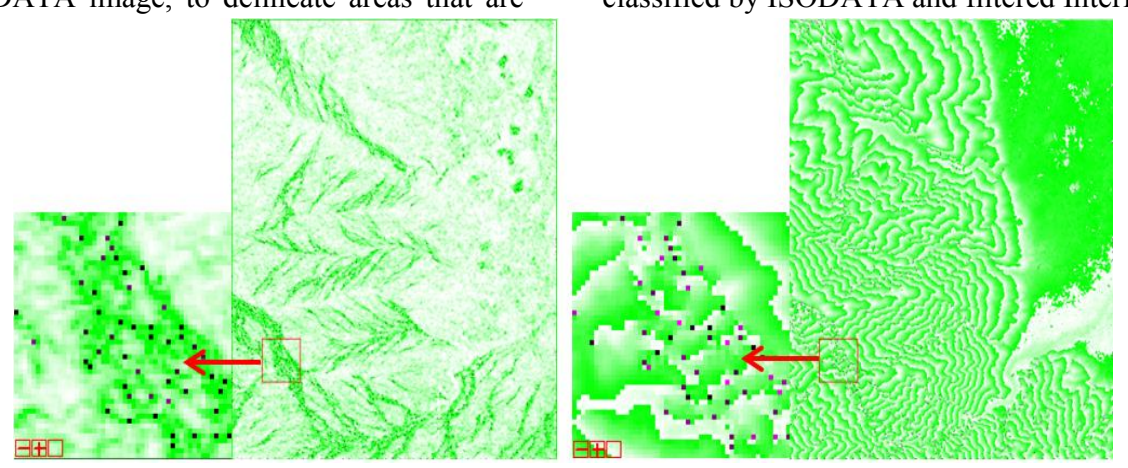

Figure10. Residues presented on ISODATA image and filtered Interferogram(Only on green site)

It is clear that the residue points occupy only the black fringes in figure 11 , which are usually very deformed terrain, where Brunch-Cut really is needed For a good unwrapping phase process.

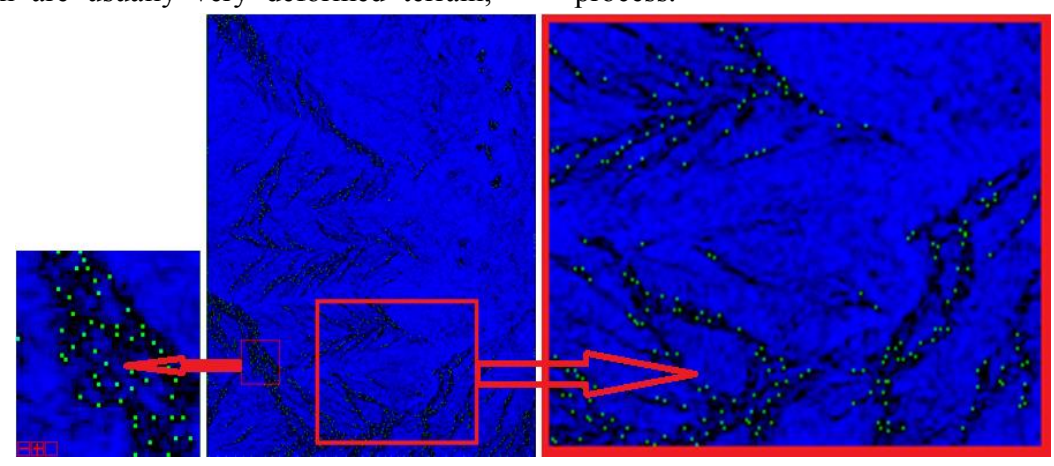


Figure 11. (R:Image by ISODATA, G:filtered B:Interferogram, Residues image).

It is really clear in Figure 12 that the brunch-cut generation by the application of the ISODATA method eliminated all black spots. That we compare the brunch-cut image before and after the application of ISODATA.

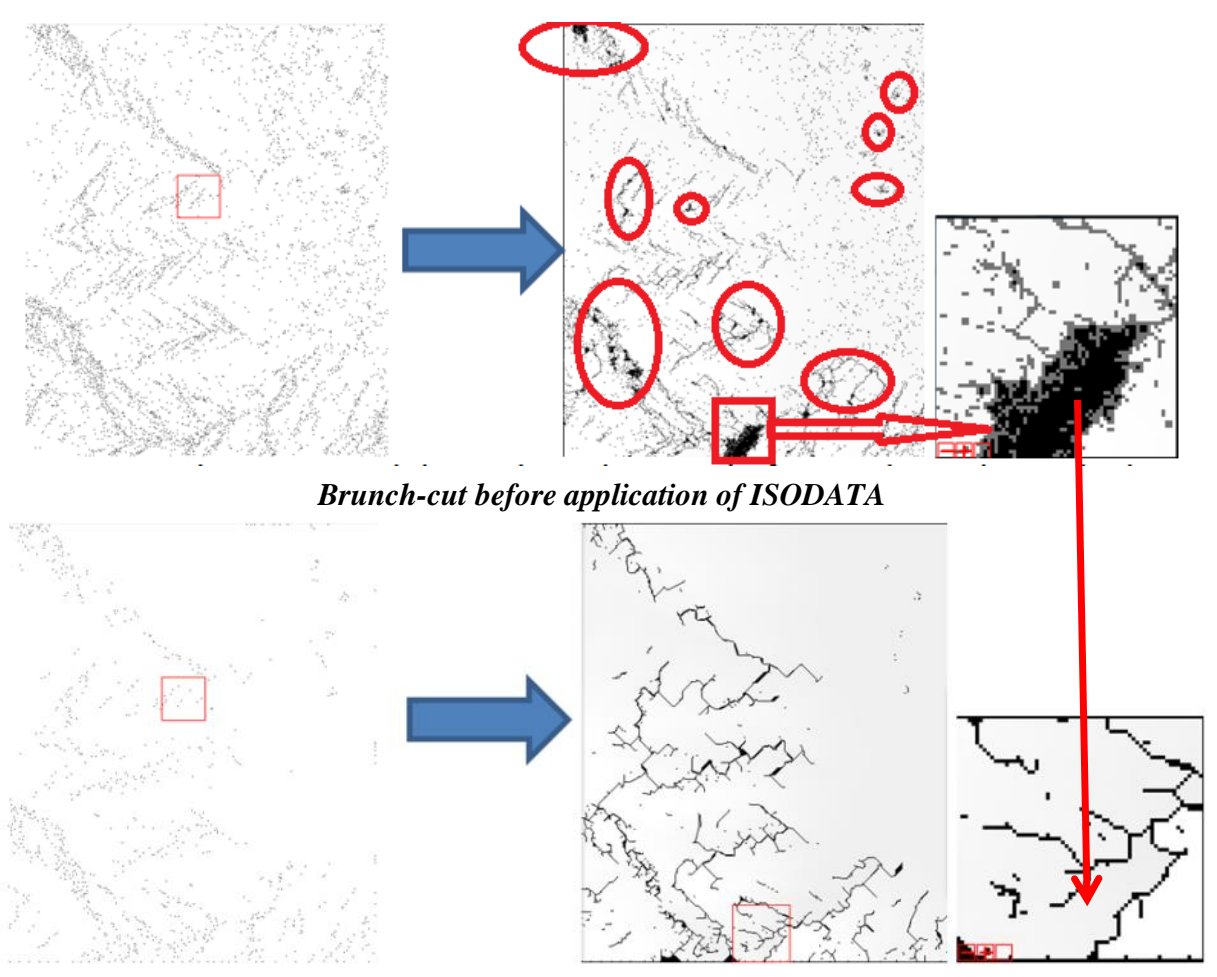

Brunch-cut after application of ISODATA

Figure 12. Comparison between compare the brunch-cut image before and after the application of ISODATA.

The following figure13 shows the DEM generated by the unwrapping phase with ISODATA method (based on KMEANS and double median filter $5 \times 5$ ). This shows a good homogeneity of the degradation of the land. 


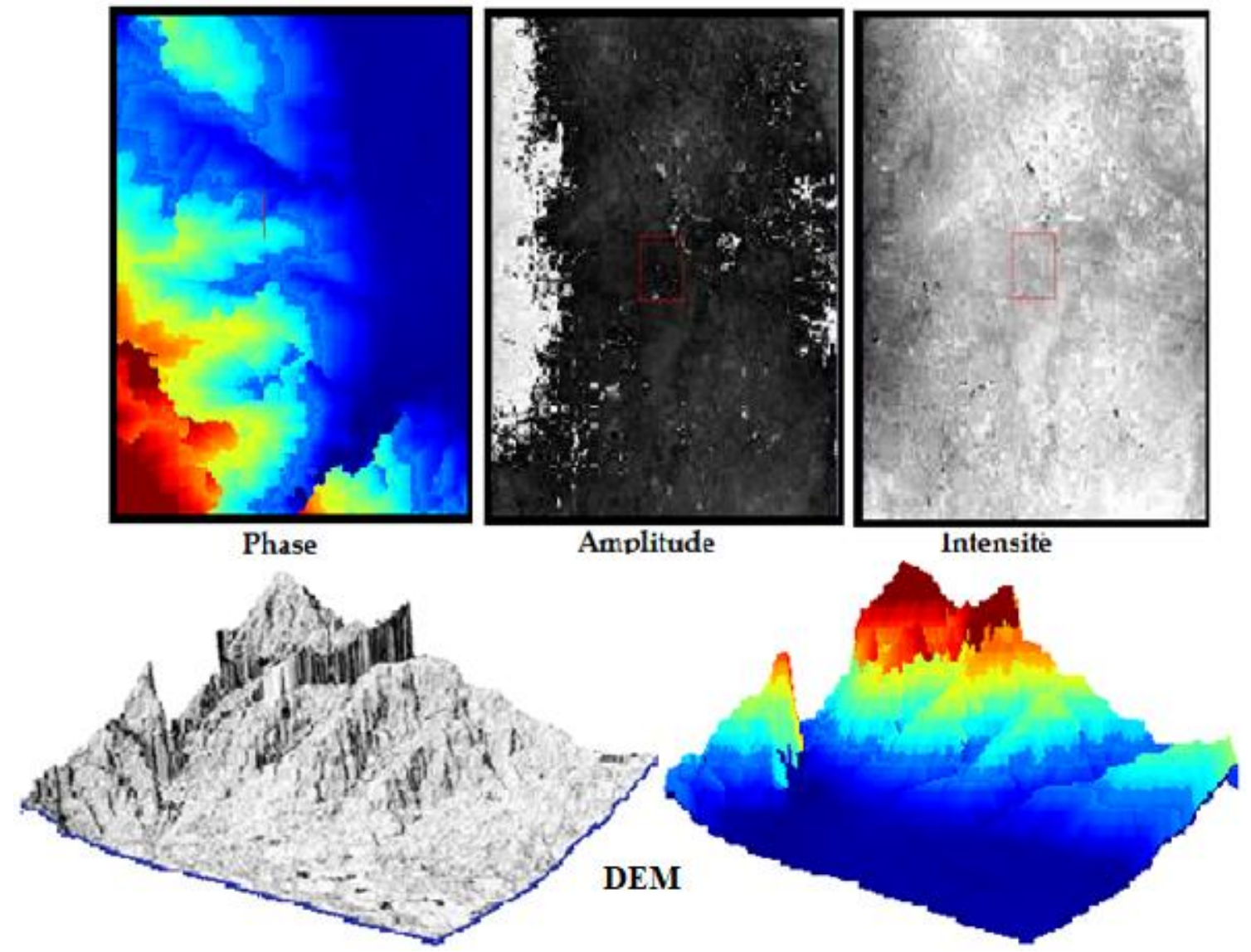

Figure 13. Final DEM with our approach for unwrapping phase.

\section{CONCLUSION:}

The phase unwrapping is a very sensitive step, that we must treat it carefully to have a good result of a DEM. The application of our approach gave better results in the different tests on the different sites. Our approach leads to applying the median filter in two passages on the interferogram without the orbital fringes. And the application of the ISODATA method based on the KMEANS on the amplitude image of the generated interferogram. The extraction of the residues from the magnitude image classified by ISODATA limits the residues on the fringes of strong deformations. In this side we trace the path of the branch-cut and on otherwise eliminates all the errors that generate black spots, this is the highlight of this approach.

\section{REFERENCES :}

[1] Argus, Donald F, Michael B Heflin, Gilles Peltzer, FrédericCrampé, and Frank H Webb. 2005. "Interseismic Strain Accumulation and Anthropogenic Motion in Metropolitan Los Angeles." Journal of Geophysical Research: Solid Earth 110 (B4).

[2] Baker, Scott, and Falk Amelung. 2012. "Top-down Inflation and Deflation at the Summit of Killauea Volcano, Hawai ' $\mathrm{i}$ Observed with InSAR.” Journal of Geophysical Research: Solid Earth 117 (B12).
[3] Chesnaud, Christophe, Philippe Réfrégier, and VladyBoulet. 1999. "Statistical Region Snake-Based Segmentation Adapted to Different Physical Noise Models." IEEE Transactions on Pattern Analysis and Machine Intelligence 21 (11): 1145-57.

[4] Graham, Leroy C. 1974. "Synthetic Interferometer Radar for Topographic Mapping."Proceedings of the IEEE 62 (6): 763 68.

[5] Kenyi, L, and H Raggam. 1997. "Accuracy Assessment of Interferometrically Derived DTMs.” In , 406:51.

[6] Massonnet, Didier, and Kurt L Feigl. 1998. "Radar Interferometry and Its Application to Changes in the Earth's Surface." Reviews of Geophysics 36 (4): 441-500.

[7] Sahraoui, O Hadj, BenaliHassaine, ChahiraSerief, and KamelHasni. 2006. "Radar Interferometry with Sarscape Software." Photogrammetry and Remote Sensing.

[8] Stilla, Uwe, UweSoergel, and Ulrich Thoennessen. 2003. "Potential and Limits of InSAR Data for Building Reconstruction in Built-up Areas." ISPRS Journal of Photogrammetry and Remote Sensing 58 (1): 113-23.

[9] Tison, Cline, Florence Tupin, and Henri Maître. 2007. “A Fusion Scheme for Joint Retrieval of Urban Height Map and Classification from High-Resolution Interferometric SAR Images." IEEE Transactions on Geoscience and RemoteSensing 45 (2): 496 505 .

\section{Creative Commons Attribution License 4.0 (Attribution 4.0 International, CC BY 4.0)}

This article is published under the terms of the Creative Commons Attribution License 4.0 https://creativecommons.org/licenses/by/4.0/deed.en US 